\title{
Level and Predicators of quality of Integrated Disease Surveillance and Response for Infectious Disease in Tigray, Northern Ethiopia: Cross- Sectional Study
}

Kiros Fenta Ajemu ( $\nabla$ kirosfenta@gmail.com )

Tigray Health Research Institute https://orcid.org/0000-0002-7608-0284

Abraham Aregay Desta

Tigray Health Research Institute

Nega Mamo Bezabih

Tigray Health Research Institute

Alemnesh Abraha Araya

Tigray Health Research Institute

Essayas Haregot Hilawi

Ottawa Hospital Research Institute

Research article

Keywords: Quality, IDSR, Infectious disease, Tigray, Northern Ethiopia

Posted Date: April 27th, 2020

DOI: https://doi.org/10.21203/rs.3.rs-24584/v1

License: (a) (1) This work is licensed under a Creative Commons Attribution 4.0 International License.

Read Full License 


\section{Abstract}

Background: The health impacts of recent global infectious disease outbreaks have demonstrated the importance of strengthening public health systems. The aim of the study was to assess the level of quality of integrated disease surveillance and response for infectious disease in public health facilities of Tigray, Northern Ethiopia.

Methods: the study was facility based cross-sectional. It was conducted from June- July 2018 in 46 health facilities. It has involved mixed method approach both quantitative and qualitative data collection methods. Donabedian input-process-output quality assessment model was used to evaluate the service. The magnitude of the association was considered at $p$-value of $\leq 0.05$ in multivariable logistic regression analysis using adjusted odds ratio (AOR) at $95 \%$ confidence interval $(\mathrm{Cl})$. Concurrently, facility surveillance officers were subjected to an in-depth interview autonomously to explore factors for good and bad service quality. Quantitative data were analyzed using SPSS version 21. Use of manual thematic approach was used for qualitative data analysis. Result: The level of the overall quality of IDSR service provision has rendered as good in 6 out of $46(13 \%)$ studied health facilities. Two third of studied health facilities were rated as good for input service quality but $34.7 \%$ for process service quality. The output service quality was two times better than the overall service quality. Being enrollment of HIT to rapid response team (AOR=7, 95\% Cl: 1.092-37.857) and accessing technical guideline to the health facility (AOR=3, 95\% Cl: 0.399-22.567) were predictor factors for facilitating overall service quality.

\section{Background}

The health impacts of recent global infectious disease outbreaks have demonstrated the importance of strengthening public health systems to better protect communities from naturally occurring and humancaused threats [1]. The International Health Regulations (2005) is a landmark legislation and testament to the renewed initiative of countries to collaboratively reduce the burden of infectious diseases [2].

Surveillance is an initiative for an on-going systematic collection, analysis, interpretation, and dissemination of health data to help and guide efficient and effective public health action for infectious disease [3]. However, surveillance is largely interpreted as a component within vertical single disease control programs in most low and middle income countries [2-3]. Evidences suggests that heavily centralized and vertical single disease control programs have been identified several drawbacks such as being inability to fulfill surveillance functions, its inadequacy to detect outbreaks timely, with little or no co-ordination to use evidence based data for decision-making, heavily autonomous in their functioning which resulted wastage of valuable resources and often not flexible[3-5].

Emergence of such challenges forced WHO to promote an integrated approach to infectious disease surveillance and response (IDSR) in the late 1998. This new approach ensure efficient use of resources, improve the use and flow of information and evidence based data thresholds to trigger alerts using local data for public health action [5-6]. For instance, Nigerian government investment to establish strong integrated disease surveillance and response for infectious disease which used a single infrastructure to 
gather information with in similar structures, personnel and processes enabled to detect rapidly and respond quickly even the worst Ebola outbreak that occurred in western Africa from 2014-2016[7-8]. Such events and achievements captured the world's attention and reinforced the importance of strengthening public health systems such as IDSR to better protect communities from naturally occurring and human-caused threats [6-7].

The IDSR frameworks provide an opportunity to leverage their limited resources to continuously improve their disease surveillance and response systems. Similarly, it enabled to generate quality and timely information that can be used to initiate epidemiological alert for efficient and effective public health actions in low income countries [9-10].

Ethiopia is among low-income countries in Africa adopted IDSR strategy in 1999. However, it had been stared implementing as it was challenged by recurrent outbreaks of malaria and measles in 2000 [11]. Similarly of acute watery diarrhea outbreaks were simultaneously reported even in the past three years in the study area in northern Ethiopia [12]. Under the umbrella of national and international recommendations, it was developed a comprehensive IDSR technical guideline that enabled health care providers to adhere with national standards and to ensure quality service provision at facility level [1314].

Due to the emergence of recurrent infectious disease outbreaks, improving quality of integrated disease surveillance and response service provision was a priority agenda in the health sector transformation plan of Ethiopia [15]. Since, program performance indicators in the national guideline were prepared in line with Donabedian input-process-output (Fig. 1) we preferred to use this quality assessment framework to evaluate the service. However, no study tried to assess level of quality of integrated disease surveillance and response services provision with respect to the three predetermined quality components. Therefore, the study aimed to assess level of quality of integrated disease surveillance and response service provision in public health facilities in Tigray, Northern Ethiopia using Donabeidan quality assessment model (Fig. 1).

\section{Methods}

\section{Study setting and period}

Tigray regional state is among the nine regional states in Ethiopia that located in the northern part of Ethiopia at distant of $805 \mathrm{KMs}$ from Addis Ababa. The region is administratively demarcated by seven zones namely East, South, South East, Western, Northwestern, Central and Mekelle. Seven zones are further sub-divided in to 52 districts (34 rural and 18 urban). The health care system in the region composed of referral hospitals (2), General hospitals (16) Primary hospitals (22), Health centers (216) and Health posts at community level (712) [12]. The study was conducted from June -July 2018. 
The aim of the study was to assess the level of quality of integrated disease surveillance and response service provision.

\section{Study design}

The study has employed facility based cross-sectional study. It has involved mixed method approach using both quantitative and qualitative data collection methods. The study employed Donabedian model of health care quality assessment framework [14]. The model was depicted in the figure below (Fig. 1).

\section{Sampling and sample size estimation}

Sampling and sample size estimation for quantitative data was done in agreement with program implementers; Tigray Regional Health Bureau and the research team members considering an earmarked budget allocated for the study. Of the total 52 districts, 14 districts were randomly selected. Accordingly, a total of 46 health facilities ( 7 hospitals and 39 health centers) were included in the study. Similarly, 23 integrated disease surveillance and response officers were subjected to an in-depth interview to obtain their views for the functioning of IDSR in the health facility.

\section{Data collection and measurements}

Data collection was conducted in line with input-process-output quality assessment dimensions of Doanabedian model [14] to evaluate the level of quality of integrated disease surveillance and response. A total of eight data collectors were recruited for data collection.

For input service quality, facility inventory was conducted using structured facility inventory check list that consists of 21 performance indicators adopted from the national guideline [13] that ensure the availability of input quality items for the functioning of IDSR. See the list variables in (additional file one).

Process service quality was assessed by using 10 process related indicators to assess the fidelity of service provider's adherence to service standards according to the national guideline. Similarly, service utilization was assessed using 5 performance indicators as an output service quality [13]. See the list variables in (additional file one).

The overall service quality was assessed by combining input, process, and output service quality components. See the list of variables used in measuring each service quality components. See the list variables in (additional file one).

Facilities were categorized rendering good service quality with respective each service quality component if the average quality performance verification score was $100 \%$ for input service quality, and $80 \%$ or more for process and output service quality components respectively [13].

Qualitative data were collected by principal investigator (Kiros Fenta) who had an experience on qualitative data collection. Integrated disease surveillance and response officers under IDSR unit were 
subjected to an in-depth interview using semi structured interview guide to explore their perception about the reasons for good and bad service quality.

\section{Operational definitions}

\section{Input dimension}

this dimension was used to assess the availability of human resources, materials, equipment, and supplies needed for integrated disease surveillance and response service provision.

\section{Process dimension}

this was used to reflect how service providers adhere to service standards during service provision of integrated disease surveillance and response.

\section{Output dimension}

this dimension in this study was used to measure the ultimate result of integrated disease surveillance and response service provision.

\section{Overall quality}

in this particular study the overall service quality of integrated disease surveillance and response was determined by considering all three service quality assessment components.

\section{Data quality assurance}

To enhance data quality, data collectors who had an experience and trained on IDSR were recruited for data collection. In addition, they were trained for two days on the nature of the tool, objective of the study and ways of approaching during an in-depth interview, and chart review. The tools were piloted and necessary modifications were made. During data collection period, there was a strict supervision and completed questionnaires were checked on a daily basis by principal investigators.

\section{Data management and analysis}

Quantitative data was entered into Epi-info version 7 and exported to SPSS version 21 statistical package for analysis. Descriptive analysis was carried out to estimate the prevalence of quality verification performance standards in respective three service quality components. Bivariate and multivariable analyses were used to assess the association between explanatory and outcome variable (level of quality). The main effect of the dependent variable was declared using enter logistic regression model. Variables which were significant at the bivariate analysis was entered to the final multivariable analysis using the Wald test ( $p$-value $<0.05$ ). The overall goodness of fit of the model was tested using HosmerLemeshow goodness-of-fit test ( $p$-value $>0.05$ ). Explanatory variables were ruled out at $p$-value of $\leq 0.25$ in bivariate analysis. The magnitude of the association was considered at $p$-value of $\leq 0.05$ in 
multivariable logistic regression analysis using adjusted odds ratio (AOR) at 95\% confidence interval (Cl) [11].

Factors for good and bad service quality were identified by analyzing qualitative data using content thematic approach [16]. This involved reading scripts several times, identifying themes and sub-themes, and grouping data according to these themes for interpretation [17]. The main study themes were; reasons for good and bad quality. All principal investigators were involved in discussions of study themes, sub-themes and interpretation of findings. The specific themes reported included case detection, quality of reporting, data analysis and coordination with respect to integrated disease surveillance and response functioning at facility level. Triangulation was made to establish a range of views related to IDSR functioning that were not captured during quantitative assessment [18].

\section{Result}

\section{Quantitative Finding}

The study was assessed based on Donabedian input-process-output service quality assessment model. All study health facilities were voluntary to participate. Of which, $94 \%$ of them were accessible with mobile network.

The study showed that the overall service quality of IDSR was rendered as good in six out of $46(13 \%)$ of studied health facilities. Input service quality was judged better than its counterparts and rated as good in two -third of studied health facilities. Process service quality was rated as good in $34.7 \%$ health facilities. Output service quality was two times better than the overall service quality (Fig. 2).

Regarding input service quality, all studied health facilities had designated IDSR service unit. Similarly, they were equipped with the necessary emergency drugs and supplies for surveillance priority disease in their underlined service unit. However; critical input related items for IDSR were missed in considerable no of studied health facilities. Only, $63 \%$ of the health facilities allocated budgets in the outbreak preparedness plan (Table 1).See all list of input item variables in (additional file one).

\section{Table (1)}

Assessment of IDSR input quality items in public health facilities, Northern Ethiopia, 2018 [N = 46] 


\begin{tabular}{|c|c|c|}
\hline Input service quality indicators & No of facility & $\%$ \\
\hline \multicolumn{3}{|l|}{ Availability } \\
\hline IDSR service unit & 32 & 69 \\
\hline Technical guide line & 36 & 78.3 \\
\hline Standard case definitions for priority disease(SCDs) & 40 & 87 \\
\hline IDSR officer & 46 & 100 \\
\hline Preparedness and response plan & 42 & 91.3 \\
\hline Patient register(IPD and OPD) & 45 & 97.8 \\
\hline \multicolumn{3}{|l|}{ Training } \\
\hline Trained IDSR officer for the past two years & 35 & 76.1 \\
\hline \multicolumn{3}{|l|}{ Coordination } \\
\hline Establishing rapid response team/taskforce & 46 & 100 \\
\hline HIT membership to RRT & 12 & 26 \\
\hline Laboratory membership to RRT & 29 & 63 \\
\hline Integration of laboratory with PHEM unit & 2 & 63 \\
\hline \multicolumn{3}{|l|}{ Resources } \\
\hline Allocating funds in the annual plan & 30 & 65 \\
\hline Emergency drugs and supplies for the past 12 months & 39 & 80 \\
\hline \multicolumn{3}{|l|}{ Registration and forms } \\
\hline Case based reporting formats & 31 & 67.4 \\
\hline AFP case investigation format & 39 & 84.8 \\
\hline Weekly reporting format & 43 & 93.5 \\
\hline Line list for case registration & 40 & 87 \\
\hline
\end{tabular}

Process service quality realized good in insignificant number of health facilities. More than three fourth of them had been practicing to report immediately reportable disease within 30 minutes based on the standard. However, most of them lack critical process quality items. No formal feedback mechanism was practiced routinely as an input for early epidemiological alert. Only, 33\% of study health facilities documented at least one written feedback in the past one year. Similarly, service provider's readiness for clinical case detection was limited. Standard case definition clearly stated for acute watery diarrhea was 
better but the worst for malaria and measles. The capacity of laboratory for specimen conformation for at least one priority disease was functional in $63 \%$ of the health facilities (Table 2). See all lists of process item variables in (additional file one).

\section{Table (2)}

Assessment of IDSR process quality items in public health facilities, Northern Ethiopia, 2018 [N = 46

\begin{tabular}{|lll|}
\hline Process service quality indicators & No of facility & $\%$ \\
\hline Case detection & 32 & 69.6 \\
\hline SCDs stated correctly for malaria & 36 & 78.3 \\
\hline SCDs stated correctly for acute watery diaharea & 27 & 58.3 \\
\hline SCDs stated correctly for measles & & 100 \\
\hline Registration & 46 & 80.4 \\
\hline Case registration in line list during an outbreak & 37 & 91 \\
\hline Notification & 42 & 84.8 \\
\hline Practice of offering IRDs to higher level within 30 minutes & & \\
\hline Have schedule for weekly reporting every Monday & 39 & 91 \\
\hline Reporting & & 63 \\
\hline Reporting weekly IDSR report to higher levels regularly & 42 & \\
\hline Case confirmation & 29 & \\
\hline Readiness for specimen collection and transportation & 35 & \\
\hline Laboratory capacity for specimen conformation & & \\
\hline Feed backs & & \\
\hline Provide at least one IDSR written feed backs to lower level & 35 & \\
\hline
\end{tabular}

According to the study finding, one fourth of the studied health facilities were achieved predetermined judgment criteria for output service quality component. Closer to half were rely on using regular trend analysis using line graph for outbreak notification but practiced only for malaria and measles.

\section{Table (3)}

Assessment of IDSR output service quality items in public health facilities, Northern Ethiopia, 2018 [N = 46] 


\begin{tabular}{|lll|}
\hline Output service quality indicators & No of facility & $\%$ \\
\hline Quality of reporting & & \\
\hline Weekly IDSR report completeness & 32 & 82 \\
\hline Weekly IDSR report timelines & 29 & 74.4 \\
\hline Decision making & & \\
\hline Summarizing IDSR data in Tables & 46 & 100 \\
\hline Preparing epidemic threshold (Measles, malaria) & 32 & 69.6 \\
\hline Perform regular trend analysis & 29 & 63 \\
\hline
\end{tabular}

Table 4: Logistic regression analysis of variables associated with overall quality of IDSR in public health facilities, Tigray, Northern Ethiopia, $2018(\mathrm{~N}=46)$.

\begin{tabular}{|c|c|c|c|c|}
\hline \multirow[t]{3}{*}{ Quality Verification indicators } & \multicolumn{2}{|l|}{$\begin{array}{l}\text { Overall } \\
\text { service } \\
\text { quality }\end{array}$} & \multirow[t]{3}{*}{$\mathrm{COR}(95 \% \mathrm{CI}$} & \multirow[t]{3}{*}{$\mathrm{AOR}(95 \% \mathrm{CI}$} \\
\hline & Yes & No & & \\
\hline & $\mathrm{n}(\%)$ & $\mathrm{n}(\%)$ & & \\
\hline \multicolumn{5}{|l|}{ HIT Member Ship to RRT } \\
\hline Yes & $8(17.4)$ & $4(8.7)$ & $0.067[0.014-0.327]^{*}$ & $7[1.092-44.857]^{* *}$ \\
\hline No & $4(8.7)$ & $30(65.2)$ & 1 & 1 \\
\hline \multicolumn{5}{|c|}{ Availability of Technical Guide Line } \\
\hline Yes & $30(65.2)$ & $2(4.3)$ & $8.333[1.376-50.468]^{*}$ & $3[0.399-22.567]^{*}$ \\
\hline No & $9(19.6)$ & $5(10.9)$ & 1 & 1 \\
\hline \multicolumn{5}{|c|}{ Trained IDSR Focal Person Availability } \\
\hline Yes & $28(60.9)$ & $4(8.7)$ & $7[1.591-30.800]^{*}$ & $1.650[0.452-6.029]$ \\
\hline No & $7(15.2)$ & $7(15.2)$ & 1 & 1 \\
\hline
\end{tabular}

Overall quality of IDSR was categorized as good or not good. Accordingly, among listed variables in the predetermined three quality components that are associated with good quality of IDSR in the bivariate analysis were HIT enrollment to rapid response team, equipping technical guide line at facility level, and providing refreshment training were fitted to multivariate logistic regression model. Finally, HIT enrollment to rapid response team ( $\mathrm{AOR}=7,95 \% \mathrm{Cl}$ : 1.092-37.857), and equipping technical guide line at facility level (AOR = 3, 95\% Cl: 0.399-22.567) were found predictor variables (Table 4).

In a multivariable analysis, enrolling health information technicians to rapid response team were associated with good quality IDSR. Health facilities having national guideline are more likely to have good quality of IDSR than those than those that don't have.

\section{Qualitative Findings}

In-depth interview of 23 IDSR focal person were recruited for qualitative data to identify their perception for good and bad service quality in each predetermined quality components. 


\section{Factors attributed to good input service quality}

\section{Regular rapid response team meeting (RRT)}

consistent with the quantitative finding, conducting regular review meeting with established rapid response team at health facility level enabled them too overcome availability related factors as described below

“..., we had been evaluating IDSR service provision regularly and identify barriers that hinder service provision early for service improvement" KII $(\neq 2)$.

\section{Factors attributed to bad input service quality}

\section{Language barrier}

majority of IDSR service providers expressed their opinion about IDSR tool preparation in English as a challenge for service providers adhere to service standards as explained below

“..., standard case definitions for IDSR were available but they were prepared in English and Amharic but not in local language "Tigrigna" as a result some health care providers couldn't understand easily for using as a reference for public health emergency management and it needs translation of local language "Tigrigna" $K I I(\neq 2)$.

"..., Even though the national guideline is available in our health facility, it was prepared in English and some health care providers can't easily understand to use it as a reference. As to me it should be available in a local language version "Tigrigna"" KII $(\neq 5)$.

\section{Factors attributed to bad process service quality}

\section{Absence of routine feedback mechanism}

majority of service providers recognized that feedback mechanism was commonly practiced orally during time of review meetings and no written feedback was practiced routinely as explained below;

"..., in our health facility, IDSR feedbacks were communicated during quarterly and half year review meetings conducted at district level but not routinely provided to use them a source of information for early outbreak emergency preparedness, response, and improving data quality. As a result, our health facilities also use similar feedback mechanism to lower level health facilities" KII ( $\neq 12)$.

\section{Factors attributed to bad output service quality}

\section{Work load}

most of the participants during an in-depth interview pointed out that lack of time due to patient load and shifting of health workers to different working units was a constraint for not performing regular trend 
analysis

not having time due patient load and working in different entry points rather than PHEM unit were main the main challenges not tracking trend analysis for early outbreak notification and detection for decision making at facility level" (KII $\neq 8$ \&10).

\section{Factors attributed to good process service quality}

\section{Health information technician RRT membership}

as described by participants of an in-depth interview, enrolling health information technicians as member of the rapid response team or IDSR taskforce enabled them to improve data utilization for decision making and explained as follows enrolling health information technicians (HIT) as a member of rapid response team or task force enables us to improve IDSR data quality such as report timelines, completeness and regular tracking of disease trend analysis for early outbreak notification and detection" (KII $\neq 10)$.

\section{Discussion}

This study addressed an important public health topic of IDSR service provision in the frame of Donabedian quality assessment model based on quality verification performance indicators in the national guideline [13]

Accordingly, $34.7 \%$ the study health facilities were judged as good in terms of process quality items but $69.6 \%$ regarding input service quality component. The two quality components were found structurally interlinked. However, input quality items were better implemented than process as similarly reported from Ghana and India $[19,20]$.

Overall, $13 \%$ of the study health facilities were rated as offering "Good quality" in both three quality assessment component which showed that the overall quality was not maintained as described in national guideline and Ethiopian health care standard for medical practices [13, 21]. However, this finding was almost two times higher than the evidence from the same study setting, Northern Ethiopia [22] which was conducted on quality of service delivery. The slight difference might be due to assessment model variation with respect to quality components in the two studies.

Two-third of the study health facilities were found equipped with the necessary input quality items as described in national guideline [13]. This implies emergency drugs and supplies, trained human power, established rapid response team, service integration, reporting formats, patient registers and allocated fund for outbreak response were readily available. However, one out of five health facilities missed the national guideline. Standard case definitions for case detection were better available (Table 2) in contrast with evidence from Malawi, Uganda and Burkina Faso [23-25] in which printed SCDs were rarely available. All available guidelines and standard case definitions were available in English version. This 
was reported as a challenge for easily using it as a reference and similarly reported from Ghana, Tanzania, Mozambique [19, 26-27] This implies technical guideline and standard case definitions with a local language "Tigrigna" version should made available at facilities level to increase health care provider's adherence to service standards for a better service quality $[19,28]$. Further, rapid response team or task force establishment was not in line with recommendation in the national guideline [13]. Some study health facilities missed key members in the RRT team such as laboratory technicians who were crucial for early notifying and detecting an outbreak and reporting to rapid response team for early epidemic preparedness and response [13]. Having such scientific realities, team establishment needs uniformity among health facilities based on the national guideline.

One from each three health facilities declared as good regarding process quality item which was much lower than a report from India and Nigeria [20,29]. This difference might be due to Nigeria strong and quality IDSR system even that enabled to control the worst Ebola outbreak in western Africa from 2014-16 [7]. Similarly, the Indian technology was very much familiar in the health sector that facilitates early notification and detection of emerging and re-emerging communicable disease [30]. The use of standard case definition was found poor for measles; nearly only half of the health care providers were being clearly defined its case definition [28] and it is comparable with evidence from India [20] but, relatively better for acute watery diarrhea and malaria. The difference might be due to health care provider's adherence to SCDs due to recurrent AWDs outbreaks happened for the past three consecutive years in the study area and similarly of malaria is an endemic disease [12]. This needs great attention by program implementers since readiness of laboratory capacity specimen conformation (63\%) at facility level was limited that might create a double burden for improving quality service. Timely communications is an important part of public health emergency preparedness and response [26, 27]. Notification of immediately reportable disease, weekly reporting, weekly report completeness (82\%) were almost comparable with predetermined targets in the national guide line (80\%) [13]. However, weekly report timeline was by far with evidence [13] but comparable with a report from [21]. Week regular data tend analysis and summarization was not practiced for priority disease at facility level based on the standard in the guideline. Only half of the study health facilities perform regular trend analysis for outbreak notification and detection. It was conducted for malaria and measles. Similar findings were obtained from Uganda, Tanzania, and Lesotho [31-33]. No formal feedback mechanism for reported data was observed. This was a challenge for early epidemiological alert and early outbreak notification. This mechanism was by far in which weekly data feedbacks were communicated using E-mail (86\%) in Sierra Leone [34]. The variation might be technological advancement in the health sector in India than Ethiopia.

\section{Conclusion}

The overall level of quality of IDSR was optimal in insignificant number of facilities. Only, $13 \%$ of health facilities recognized achieving the overall service quality based on three quality components. To realize the current aspired level of service quality in the country's health sector transformation plan, the three quality components will be kept on eye side by side during service mentoring. This is because the three quality components are interrelated and the effect of one component had an impact on the other [14]. 
Having this reality, persistent effort in view of Donabedian's theoretical framework will also improve the overall service quality. Similarly, enrolling health information technician as member of rapid response team and accessing national technical guideline at facility level improve service quality.

\section{Study limitations}

Donabedian model of quality assessment was used in the study. This model had its own drawbacks that considered only linear assumption that do not infer casual relationships. Similarly, Hawthorn or social desirability biases might be happened during an in-depth interview. In addition, rigor statistical test was not done since it was program based process evaluation

\section{Abbreviations}

PHEM: Public Health Emergency Management; IDSR : Integrated Disease Surveillance and Response; RRT: Rapid Response Team; HIT: Health Information Technicians; WHO: World Health Organization; AWD: Acute Watery Diarrhea; IRDs: immediately reportable disease; KII: Key informant interviews; SCDs: standard case definitions

\section{Declarations}

\section{Ethical approval and consent to participate}

The study protocol was reviewed and approved by the Institutional Review Board of Tigray Health Research Institute (institusionsl.review.board.thri@gmail.com) with a reference and approval no (THRI-IRB 0040/2018). Permission was also received from the Tigray Regional Health Bureau in written letter. Data collection was conducted only after an informed consent was given in written form to service providers.

\section{Consent for publication}

Not applicable

\section{Availability of data and materials}

The datasets used and/or analyzed for the study were available from the corresponding author on reasonable request.

\section{Competing interests}

The authors declare that they have no competing interests.

\section{Funding}

Government research grant was provided to Tigray Health Research Institute with a grant number [THRI341/02/6223]. This grant was allocated for IDSR quality service evaluation in public health facilities. The 
funders had no role in study design, data collection, analysis and interpretation, decision to publish, or preparation of the manuscript.

\section{Authors' contributions}

$\mathrm{KF}, \mathrm{NM}, \mathrm{AbA}$, AlA, EH designed the study; KF, data analysis, interpretation, and preparing the first draft of the manuscript. All authors were involved in commenting, revising, and approving the final version of the manuscript.

\section{Acknowledgments}

The authors would like to thank Tigray Regional Health Bureau, respective study health facilities and study team for their support and contribution to the study. The authors are also grateful to the study participant

\section{Author's details}

1. Kiros Fenta (MPH: Researcher at Tigray Health Research Institute, Mekelle, Tigray, Ethiopia. E-mail: kirosfenta@gmail.com Mobile №: +251-9-35532829

2. Abraham Aregay(MSC, Researcher at Tigray Health Research Institute, Mekelle, Tigray, Ethiopia. Email:abaregaydesta@gmail.com, Mobile No: +251-9-23454554

3. Nega Mamo (MSC, Researcher at Tigray Health Research Institute, Mekelle, Tigray, Ethiopia. Email:negamamo40@gmail.com Mobile No: +251-9-14756767

4. Alemnesh Araya (MPH: Researcher at Tigray Health Research Institute, Mekelle, Tigray, Ethiopia. Email: alemneshab2017@gmail.com, Mobile №: +251-9-14759940

5. Essayas Haregot (PhD): Director General at Tigray Health Research Institute, Mekelle, Tigray, Ethiopia.Email:esayasharegot@gmail.com, Mobile №: +251-9-29103558

\section{References}

1. World Health Organization. Health Emergencies Programme in the African Region: Annual Report Regional Office for Africa; 2016. http:// www.afro.who.int/fr/node/8317.

2. World Health Organization. International health regulations. Third Edition, 2005.

3. Perry HN, McDonnell SM, Alemu W, et al. Planning integrated disease surveillance and response system: a matrix of skills and activities. BMC Med. 2007;5:24. doi:10.1186/1741-7015-5-24.

4. Kasolo F, Yoti Z, Bakyaita N, Gaturuku P, Katz R, et al. IDSR as a platform for implementing IHR in African countries. Biosecur Bioterror. 2013;11:163-9.

5. World Health Organization, Regional Office for Africa. Mapping risks and the distribution of epidemics in the WHO African region. 2016; a technical report; 2016.

6. WHO. CDC. Technical Guidelines for Integrated Disease Surveillance and Response in the African Region. 2nd ed: World Health Organization, Regional Office for Africa; 2010. 
7. Heymann DL, Chen L, Takemi K, et al. Global health security: the wider lessons from the West African Ebola virus disease epidemic. The Lancet. 2015;385:1884-901.

8. Nsubuga P, Nwanyanwu O, Nkengasong JN, et al. Strengthening public health surveillance and response using the health systems strengthening agenda in developing countries. BMC Public Health 10, S5 (2010) doi:10.1186/1471-2458-10-S1-S5.

9. World Health Organization. Protocol for the assessment of national communicable disease surveillance and response systems: guidelines for assessment teams. 2001.

10. Groseclose SL, Buckeridge DL. Public health surveillance systems: recent advances in their use and evaluation. Annu Rev Public Health. 2017;38:57-79.

11. "Ethiopian Field Epidemiology Training Program (EFETP)

Zemelak E. "Ethiopian Field Epidemiology Training Program (EFETP) Compiled Body of Works in Field Epidemiology," Master of Public Health in Field Epidemiology, School of Public Health, Addis Ababa University Addis Ababa University May, 2014.

12. The Government of Tigray National Regional State. Bureau of Health Tigray Regional Health Bureau 2008 EFY Annual Profile.

13. The Federal Democratic Republic Ethiopia. Ministry of Health (FDRE-MOH): Public Health Emergency Management Guideline, 2012.

14. Donabedian A. (1988) the Quality of Care: How Can It Be Assessed? Journal of the AmericanMedicalAssociation,260,17431748.http://dx.doi.org/10.1001/jama.1988.03410120089033.

15. FMOH. Health Sector Transformation Plan (HSTP) 2015/16-2019/20. Addis Ababa: Federal Ministry of Health; 2015.

16. Graneheim UH. L.B.Q.c.a.i.n.r.c., procedures and measures to achieve trustworthiness. Nurse Educ Today. 2004;24(2):105-12.

17. a rev

Synthesizing qualitative and quantitative evidence Dixon-Woods M, Agarwal S, Jones D, Young B, Sutton A. Synthesizing qualitative and quantitative evidence: a rev.

18. Tong A, Sainsbury P, Craig J. Consolidated criteria for reporting qualitative research (COREQ): a 32item checklist for interviews and focus groups. Int J Qual Health Care. 2007;19(6):349-57.

19. Adokiya MN, Awoonor-Williams JK, Barau IY, et al. Evaluation of the integrated disease surveillance and response system for infectious diseases control in northern Ghana. BMC Public Health. 2015;15:75. doi:10.1186/s12889-015-1397-y.

20. Phalkey RK, Shukla S, Shardul S, et al. Assessment of the core and support functions of the Integrated Disease Surveillance system in Maharashtra, India. BMC Public Health. 2013;13:575. doi:10.1186/1471-2458-13-575.

21. Ethiopian Standards Agency (ESA) and Technical Committee for Medical Care Practices (TC90). Ethiopian standard: health center requirements first ed. Vol. ES3611. Addis Ababa: Ethiopian Standards Agency (ESA); 2012. 
22. isseha G, Berhane $Y$, Worku A. Quality of intrapartum and newborn care in Tigray, Northern Ethiopia. BMC Pregnancy Childbirth. 2019;19:37. doi:10.1186/s12884-019-2184-z.

23. Joseph Wu T-S, Kagoli M, Kaasbøll JJ, Bjune GA. Integrated Disease Surveillance and Response (IDSR) in Malawi: Implementation gaps and challenges for timely alert. PLoS ONE. 2018;13(11):e0200858. https://doi.org/10.1371/journal.pone.0200858.

24. Kihembo C, Masiira B, Nakiire L, et al. The design and implementation of the re-vitalised integrated disease surveillance and response (IDSR) in Uganda, 2013-2016. BMC Public Health. 2018;18:879. doi:10.1186/s12889-018-5755-4.

25. Somda ZC, Perry HN, Messonnier NR, Djingarey MH, Ki SO, Meltzer MI. Modeling the CostEffectiveness of the Integrated Disease Surveillance and Response (IDSR) System: Meningitis in Burkina Faso. PLoS ONE. 2010;5(9):e13044. https://doi.org/10.1371/journal.pone.0013044.

26. Gueye D, Banke KK, Mmbuji P. Follow- up monitoring and evaluation of integrated disease surveillance and response in Tanzania; 2006.

27. Mozambique MOH: Assessment of Epidemiological Disease Surveillance System in Mozambique. Ministry of Health and the World Health Organization; 2006.

28. 10.1186/s12889-018-5028-2

Toda M, Zurovac D, Njeru I, Kareko D, Mwau M, Morita K. Health worker knowledge of Integrated Disease Surveillance and Response standard case definitions: a cross-sectional survey at rural health facilities in Kenya. BMC Public Health. 2018; 18(1):146. Published 2018 Jan 17. doi:10.1186/s12889-018-5028-2.

29. Isere EE, Fatiregun AA, Ajayi IO. An overview of disease surveillance and notification system in Nigeria and the roles of clinicians in disease outbreak prevention and control. Niger Med J. 2015;56(3):161-8. doi:10.4103/0300-1652.160347.

30. kant L, Krishnan SK. Information and communication technology in disease surveillance, India: a case study. BMC Public Health 10, S11 (2010) doi:10.1186/1471-2458-10-S1-S11.

31. Lukwago L, Nanyunja M, Ndayimirije N, Wamala J, Malimbo M, Mbabazi W, Gasasira A, Nabukenya IN, Musenero M, Alemu W, et al: The implementation of Integrated Disease Surveillance and Response in Uganda: a review of progress and challenges between 2001 and 2007. Health Policy Plan 2012.

32. Mghamba JM, Mboera LEG, Krekamboo W, Senkoro KP, Rumisha SF, Shayo EH, Mmbuji P. Challenges of implementing an Integrated Disease Surveillance and Response strategy using the current Health Management Information System in Tanzania. Tanzania Health Bulletin. 2004;6(2):57-63.

33. Lesotho $\mathrm{MOH}$ : Implementation of Integrated Disease Surveillance and Response in Lesotho. Ministry of Health and Family Welfare and World Health Organization; 2004.

34. Njuguna C, Jambai A, Chimbaru A, et al. Revitalization of integrated disease surveillance and response in Sierra Leone post Ebola virus disease outbreak. BMC Public Health. 2019;19:364. doi:10.1186/s12889-019-6636-1. 
Figures

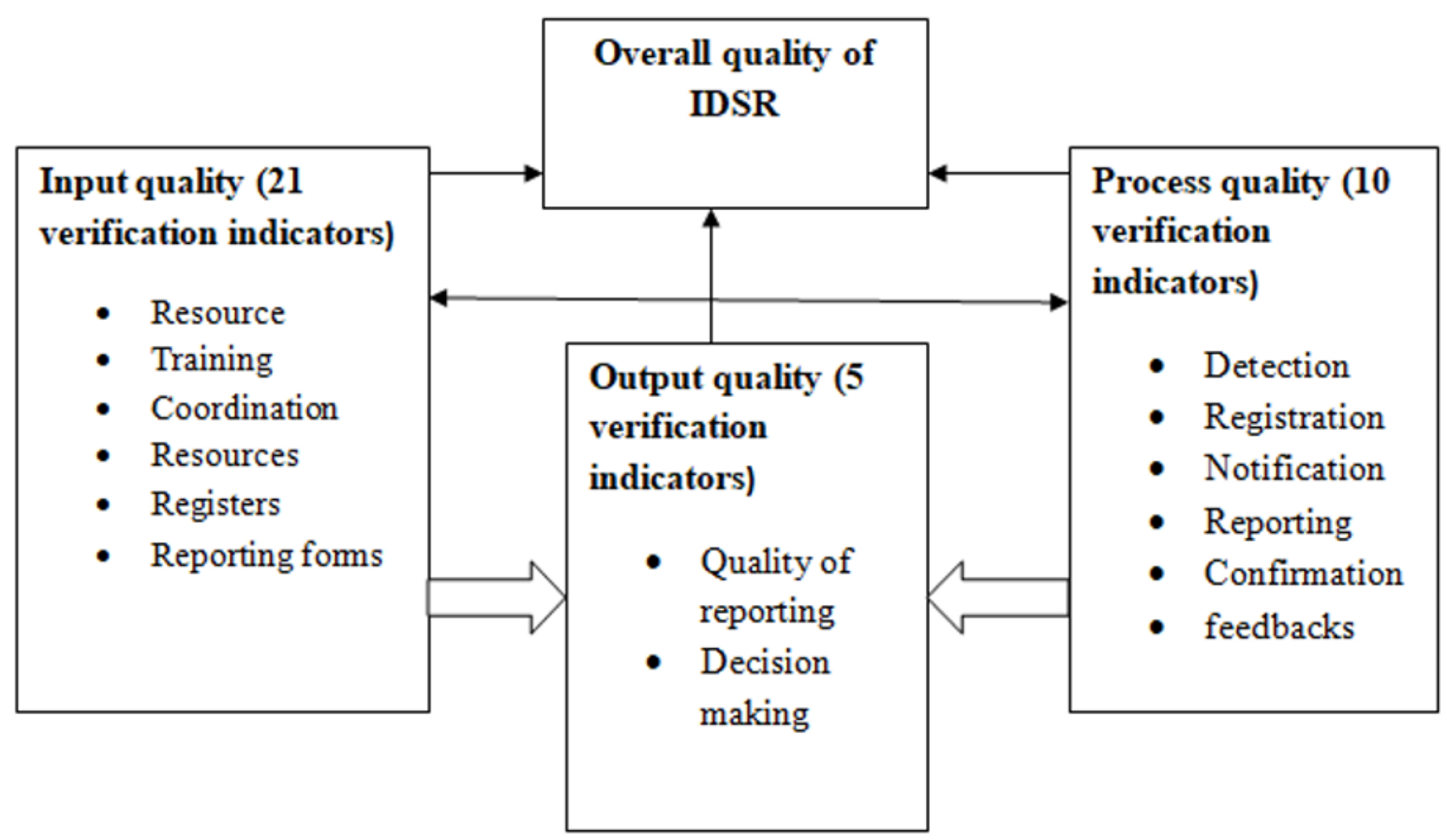

Figure 1

Conceptual frame work for assessing quality of IDSR adopted from [13] 


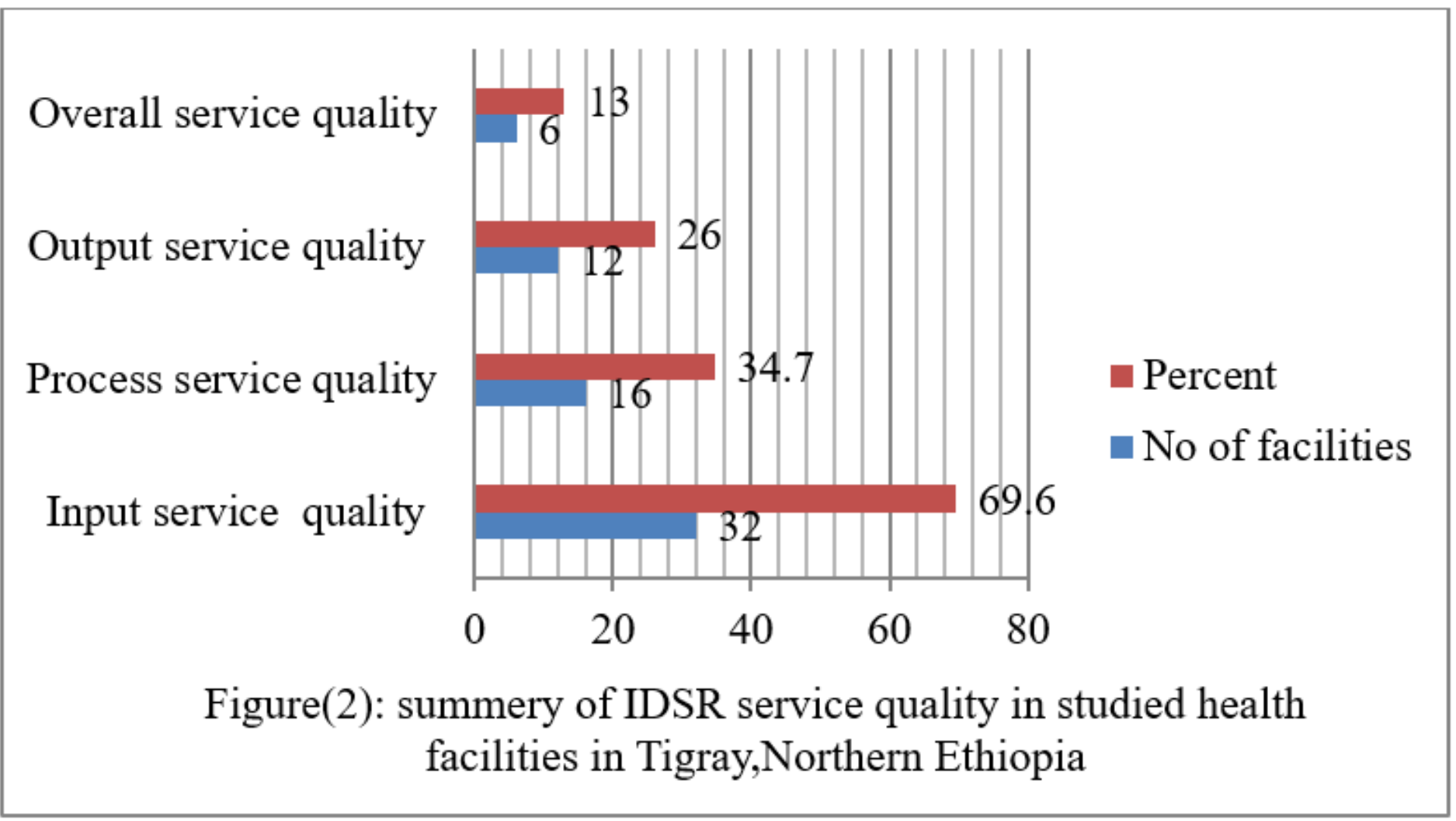

Figure 2

Summery of IDSR service quality in studied health facilities in Tigray, Northern Ethiopia

\section{Supplementary Files}

This is a list of supplementary files associated with this preprint. Click to download.

- XLfileDetailqualityscore.xlsx 